\title{
Quantifying morphological variation in the Castilleja pilosa species complex (Orobanchaceae)
}

\author{
Sarah J. Jacobs ${ }^{\text {Corresp., Equal first author, 1, 2, 3 }}$, Sarah Herzog ${ }^{\text {Equal first author, 1,2 }}$, David C. Tank ${ }^{\text {1, 2, } 3}$ \\ ${ }^{1}$ Stillinger Herbarium, University of Idaho, Moscow, Idaho, United States \\ 2 Biological Sciences, University of Idaho, Moscow, Idaho, United States \\ 3 Institute for Bioinformatics and Evolutionary Studies (IBEST), University of Idaho, Moscow, Idaho, United States \\ Corresponding Author: Sarah J. Jacobs \\ Email address: sarahjjacobs@gmail.com
}

Robustly delimited species are of paramount importance, the identification of which relies on our ability to discern boundaries between one species and the next. This is not difficult to do when species are very distinct from one another. However, in recently evolved lineages where putative species may have relatively few diagnostic features (e.g., species complexes composed of very similar species, the boundaries between which are often unclear), defining species boundaries can be more challenging. Hence, the field of species delimitation has widely advocated the use of multiple lines of evidence to delimit species, particularly in species complexes. Excessive taxonomic confusion, often the result of species descriptions that shift through time (e.g., during revisionary work and regional treatments), can further complicate the search for diagnostic features in species complexes. Here, as a first step in robustly delimiting species boundaries, we quantify and describe morphological variation in the Castilleja pilosa species complex. We first infer the morphospace of the species complex and use fuzzy-clustering techniques to explore the morphological variation in the system. Next, we hypothesize the position of type specimens within that morphospace. In so doing, we aim to visualize the impact that regional treatments have had on the conceptualization of taxa through time. We find that there is limited morphological variation among members of this complex, and we determine that the morphological concept of these species have shifted through time and are no longer accurately represented by species descriptions. 


\section{Quantifying morphological variation in the Castilleja pilosa species complex}

\section{2 (Orobanchaceae)}

3

4 Sarah J. Jacobs ${ }^{1,2,3}$, Sarah Herzog ${ }^{1,2}$, David C. Tank ${ }^{1,2,3}$

5

$6 \quad{ }^{1}$ Biological Sciences, University of Idaho, Moscow, Idaho, USA

$7 \quad 2$ Stillinger Herbarium, University of Idaho, Moscow, Idaho, USA

$8{ }^{3}$ Institute for Bioinformatics and Evolutionary Studies (IBEST), University of Idaho, Moscow, 9 Idaho, USA

10

11 Corresponding Author:

12 Sarah J. Jacobs

13875 Perimeter Drive MS 3051, Moscow, Idaho, USA 83844-3051

14 Email address: sarahjjacobs@gmail.com 
16

17

\section{Abstract}

Robustly delimited species are of paramount importance, the identification of which relies on our ability to discern boundaries between one species and the next. This is not difficult to do when species are very distinct from one another. However, in recently evolved lineages where putative species may have relatively few diagnostic features (e.g., species complexes composed of very similar species, the boundaries between which are often unclear), defining species boundaries can be more challenging. Hence, the field of species delimitation has widely advocated the use of multiple lines of evidence to delimit species, particularly in species complexes. Excessive taxonomic confusion, often the result of species descriptions that shift through time (e.g., during revisionary work and regional treatments), can further complicate the search for diagnostic features in species complexes. Here, as a first step in robustly delimiting species boundaries, we quantify and describe morphological variation in the Castilleja pilosa species complex. We first infer the morphospace of the species complex and use fuzzy-clustering techniques to explore the morphological variation in the system. Next, we hypothesize the position of type specimens within that morphospace. In so doing, we aim to visualize the impact that regional treatments have had on the conceptualization of taxa through time. We find that there is limited morphological variation among members of this complex, and we determine that the morphological concept of these species have shifted through time and are no longer accurately represented by species descriptions.

\section{Introduction}

Because they provide the basis for the recognition of one of the primary units of biodiversity, the species, classifications are the cornerstone of the biodiversity sciences. As such, classifications 
39 are vital to our understanding of biodiversity and the process of speciation. Therefore, the careful 40 and robust delimitation of species is imperative. Species delimitation relies on our ability to

41 define boundaries between one population and the next. Historically, this has been done using 42 morphological evidence (Sneath and Sokal 1974), ecological evidence (Van Valen 1976), and more recently, in light of technical and analytical advances, molecular evidence (Baum and

44 Shaw 1995). Each criterion has limitations for being widely applied across the tree of life (De 45 Queiroz 2007), and no one criterion has been universally applied to defining species boundaries 46 (De Queiroz 2009). Instead, there has been a movement to include multiple lines of evidence in 47 the delimitation of species (e.g., Padial et al. 2010; Schlick-Steiner et al. 2010; Carstens et al. 48 2013; Dejaco et al. 2016; Freudenstein et al. 2016). In addition to describing newly discovered 49 species, species delimitation methods are often applied to existing classifications where species boundaries are poorly defined and/or sample assignment to species is difficult (e.g. Barley et al. 2013; Giarla et al. 2014). In these cases, species delimitation is used in a validation context

52 (where taxonomic boundaries are validated-i.e., individuals are assigned to a group a priori

53 (Ence and Carstens 2010)) and attempts to clarify species boundaries and which lines of 54 evidence (morphological, ecological, molecular) do and do not describe species. Traditionally, species have been described and established, in part, by designating a type

specimen. From the type collection and other specimens examined during species discovery, morphological, ecological, and distributional traits are used to create a species description, providing a central reference point or general conceptualization of the new species, around which some amount of variation occurs (Fig. 1). However, the characteristics of this variation (the amount, the direction, etc.) are not static, and additional collections assigned to a species can shift the conceptual boundaries of the species, in particular how this is applied on-the-ground. 
62 For example, regional and floristic studies can result in treatments and species descriptions that

63 incorporate variation observed in the field on a local scale. Revisionary work, typically

64 happening at a broader scale (e.g. the Flora of North America), often recognizes overlapping

65 variation between similar species and synonymizes names where appropriate. As a result, there

66 can be a shift of species boundaries and known variation. In essence, these shifts can inflate or

67 deflate the taxonomic concept of an entity, sometimes greatly outside the realm of its original

68 description. Taxonomic drift such as this can result in a species description that no longer

69 represents the range of described variation for a species, and instead only represents a portion of 70 that variation (Fig. 1).

71 Often, the species involved in these taxonomic fluctuations are characterized as species

72 complexes (i.e., groups of species that are difficult to distinguish from one another), and are

73 already known to have overlapping variation that is difficult to classify. The shifting of

74 recognized and ascribed variation through time and across treatments can further increase the

75 fuzziness of species boundaries, making the identification of unknown individuals (and therefore

76 the usefulness of the classification) even more difficult. This is further complicated when an

77 unknown comes from a geographic boundary (or, conversely, one that is widespread but has

78 varieties that occur in geographically restricted areas), served by two or more regional or

79 localized treatments that have varying interpretations of variation within a taxon. This requires

80 choices to be made by the identifier in preferring one treatment to another when treatments are in

81 conflict (e.g., one treatment recognizes varieties, while another does not). Cases such as these-

82 species complexes with a great deal of taxonomic confusion — are good targets for robust species

83 delimitation. By clarifying and determining which lines of evidence distinguish species,

84 classifications can be updated to reflect more accurate estimates of species boundaries. 

notoriously difficult, particularly in the field. These difficulties largely stem from a nearly

87 continuous range of variation both within and across taxonomic boundaries (Cronquist et al.

88 1984). The source of this morphological continuity is likely a combination of the young age of

89 the lineage, the widespread and highly variable instances of polyploidy, and interspecific gene

90 flow when species co-occur (Heckard and Chuang 1977; Tank et al. 2008; Tank et al. 2009).

91 This means that most often the characters that diagnose species are slight and often overlapping.

A good example of these difficulties can be found in the Pilosae alliance. Composed of approximately eight taxa and several varieties (Castilleja arachnoidea Greenm., C. cinerea A.

Gray, C. nana Eastw., C. pilosa (S. Watson) Rydb. (with three named varieties: var. pilosa, var. longispica (S. Watson) N.H. Holmgren, and var. steenensis (Pennell) N.H. Holmgren), C. praeterita Heckard \& Bacig., C. rubida Piper, C. salsuginosa N.H. Holmgren, and C. schizotricha Greenm.), members of this group have tubular flowers with less-showy corollas, short beaks, and a pouchy, lower corolla lip that has somewhat petaloid teeth (Fig. 2b) (Cronquist et al. 1984; Hitchcock et al. 1984; Wetherwax et al. 2012). This morphological alliance is unique among most Castilleja alliances in that it is composed of perennial diploids, with no documented polyploidy (Heckard and Chuang, 1977).

Within the alliance, two widespread species are especially difficult to distinguish, particularly in the field_Castilleja pilosa and Castilleja nana. These taxa compose the core members of the pilosa species complex. C. pilosa is composed of three taxonomically recognized varieties, distinguished primarily by geography, in addition to slight variations in a suite of morphological characters. Castilleja pilosa var. pilosa is found in the Sierra Nevada, north and east into Oregon; Castilleja pilosa (S.Watson) Rydb. var. steenensis (Pennell) N.H.Holmgren is 
108 endemic to the high ridges of Steens Mountain in southeastern Oregon; Castilleja pilosa

109 (S.Watson) Rydb. var. longispica (A.Nelson) N.H.Holmgren occurs in the southern half of

110 Idaho, east into western Wyoming and Montana, and has a disjunct population in northern Idaho.

111 These varieties are distinguished by calyx length, herbage pubescence, elevation, and geographic

112 position (Fig. 2a) (Cronquist et al. 1984). Castilleja nana occurs throughout the central and

113 southern Sierra Nevada range of eastern California and extends eastward on the high ridges of

114 Nevada's basin and range topography. Castilleja nana is primarily distinguished from C. pilosa

115 by elevation, occuring between 2400 and 4200 meters, while $C$. pilos $a$ is found primarily at

116 lower elevations between 1200 and 3400 meters. Additionally, C. nana is often a smaller plant

117 with decumbent branches and smaller features (Fig. 2a).

118 When Castilleja pilosa and Castilleja nana occur in sympatry and at the same elevation,

119 it is often quite difficult to distinguish the two species. Additionally, many of the members of

120 this complex occur across geographic and political boundaries and are represented in multiple,

121 overlapping regional and floristic treatments (Cronquist et al. 1984; Hitchcock et al. 1984;

122 Wetherwax et al. 2012). Subsequently, there has been a great deal of taxonomic confusion,

123 demonstrated by the number of synonyms associated with C. pilosa and C. nana. Several of

124 these incorporations are based on collections made in the Sierra Nevada where both species

125 occur in sympatry, as well as northern California at the border with Oregon and Nevada. These

126 regions also lie at the boundary between the Great Basin, the Pacific Northwest, and the Sierra

127 Nevada and California floristic province, where a great deal of taxonomic work has been done.

128 The taxonomic confusion in this group could be the result of any of the following factors: the

129 young age of the lineage, the propensity for gene flow when species are sympatric, little to no

130 morphological distinction between species, and/or the absence of species in the complex (i.e., the 
131 entire complex is actually a single lineage). As such, this complex is in great need for robust

132 species delimitation.

133 Here we begin this process by quantifying morphological variation in the complex and

134 assessing its correlation (or not) with the current taxonomy. By sampling many populations

135 across the known ranges of these entities, identifying them using regional treatments, and

136 measuring and analyzing a suite of morphological traits, we test the assumption that there are

137 morphological clusters that correspond to taxonomic entities. We perform principal coordinate

138 analyses to understand the position of individuals in morphospace, and then apply a non-

139 hierarchical clustering method to assess the signal of morphological similarity that exists among

140 these entities. In this way, we aim to quantify and begin to characterize the morphological

141 variation in this species complex, information that will ultimately become part of a robust

142 delimitation of species boundaries in this group.

143

144 Materials \& Methods

145 Sampling and Range Estimation. Both mounted and unmounted collections of Castilleja

146 pilosa var. pilosa, C. pilosa var. longispica, C. pilosa var. steenensis, and C. nana were

147 examined for this study, with emphasis placed on representing the hypothesized distributional

148 ranges of these taxa. Prior to measurement, all collections were identified using the primary

149 literature currently available — regional floras and treatments ((Wetherwax et al. 2012)

150 California; (Hitchcock et al. 1984), Oregon, Idaho, and adjacent Montana and Wyoming;

151 (Cronquist et al. 1984), Great Basin). Species ranges were estimated based on loan material and

152 specimen label data accessed through regional databases (Consortium of Pacific Northwest

153 Herbaria (pnwherbaria.org); Southwest Environmental Information Network (SEINet; 
154 swbiodiversity.org); University and Jepson Herbaria Specimen Portal

155 (webapps.cspace.berkeley.edu); New York Botanical Garden (NYBG; nybg.org); Rocky

156 Mountain Herbarium (RM; rmh.uwyo.edu)). Latitude and longitude were taken directly from

157 collection labels, when available. In some cases, coordinates were not provided on the collection

158 label, in which case they were estimated by hand based on locality information provided by the

159 collector. For specimens whose identification we did not confirm (i.e., specimens not on loan),

160 we only considered collections or identifications determined by collectors that we considered to

161 have extensive expertise in Castilleja identification. All sampling information, including

162 coordinates and voucher locations, can be found in the Supplemental Information (Table S1).

163 Morphological Measurements. We used a combination of continuous and categorical

164 traits to characterize morphology. These traits are known to be taxonomically informative, and

165 are widely used to identify and distinguish Castilleja species (Cronquist et al. 1984; Hitchcock et

166 al. 1984; Chuang and Heckard 1991; Hersch-Green and Cronn 2009) (Table 1). Specimens were

167 chosen for data collection based on the overall condition of the collection and maturity of the

168 plant when it was collected, preferring specimens as close to peak flower as possible. Multiple

169 stems within each collection were measured in order to record a complete set of measurements

170 for each collection. Floral measurements were taken from dissected flowers rehydrated with

171 Pohl's solution (Pohl 1965). Flowers at peak maturity were identified, removed from the

172 indeterminate inflorescence, and saturated with Pohl's solution for five minutes. The bract,

173 calyx, and corolla were separated from one another, and measurements taken from the dissected

174 tissues (Fig. 2b). Habit, inflorescence, and leaf characters were taken from the specimen without

175 further dissection; surface textures were taken from stem midway between the inflorescence and

176 the base of the plant. 

nana $(\mathrm{n}=50)$, C. pilosa var. longispica $(\mathrm{n}=34)$, C. pilosa var. pilosa $(\mathrm{n}=76)$, and C. pilosa var. steenensis $(\mathrm{n}=11)$. Several continuous variables were used in the auto-calculation of additional continuous variables, thus creating a composite variable (Table 1, characters 18, 27, and 28). To avoid pseudo-replication of traits in the dataset, we removed the component traits (Table 1, characters $16,17,21,22,23$, and 27 ), leaving only the composite variables in the dataset, resulting in thirteen quantitative characters. Nine categorical characters were recorded from the same 171 collections (Table 1). Three of these characters did not vary across individuals and were removed from the dataset (Table 1, characters 24, 25, and 26), leaving a total of six qualitative characters included in the analyses.

Data Preparation and Quantification of Morphological Variation. When present, raw measurements from different stems of the same collection were combined to produce an average measurement for each individual for each trait examined. Individuals with missing data for any of the traits measured (indicating the tissue was unavailable for sampling, a total of 60 collections) were removed from downstream analyses. We identified possible outliers in the dataset by calculating the multivariate normal density function of all continuous variables using the stats package in R (R Core Team 2016), resulting in the pruning of 10 collections. Continuous variables were log transformed, and presence/absence data were coded as binary variables.

Principal Coordinate Analysis. To represent the morphological similarity in our dataset, we applied a metric, multidimensional scaling approach that positions each individual in a reduced dimension morphospace, preserving the distance relationship between individuals as well as possible (Gower 1966). Because the categorical variables that we measured are 
200 taxonomically diagnostic, it was important to include them in a quantification of morphospace in 201 this species complex. We performed a principal coordinate analysis (PCoA), which can handle 202 both quantitative and qualitative data by using measures of (dis)similarity calculated from mixed 203 variables (Gower 1966; Legendre and Legendre 1998). We calculated a dissimilarity matrix 204 based on our log-transformed continuous variables, our nominal categorical variables, and our 205 symmetric dichotomous variables, using Gower's dissimilarity coefficient (Gower 1971), as 206 implemented using the daisy function in the R package cluster (Maechler et al. 2016). We then 207 performed PCoA on the dissimilarity matrix using the function pcoa in the R package ape 208 (Paradis et al. 2004). PCoA can sometimes result in negative eigenvalues when dealing with non209 Euclidean distance measures (as we are doing here). As such, we used the Cailliez correction 210 (Cailliez 1983), where a constant is added to each original measure of dissimilarity (except the 211 diagonals). Because PCoA is based on a pairwise distance matrix, there are approximately as 212 many dimensions as there are pairwise comparisons, and they are ordered by their eigenvalues. 213 By plotting each individual at the first two to three principal coordinates, we can represent the 214 best possible Euclidean approximation of the morphological distance between them (Gower 215 1982).

216 Fuzzy Clustering. To explore and describe the signal of morphological similarity that we 217 have quantified, we apply a clustering technique that can accommodate situations where cluster 218 boundaries may not be clear-cut. Fuzzy clustering (Dunn 1976; Kaufman and Rousseeuw 2005) 219 is a 'soft' approach to clustering where individuals are assigned a probability of membership (the 220 coefficient of membership) to each recovered cluster; this is in contrast to 'hard' clustering

221 where an individual is assigned to a single cluster only. The benefit of this type of clustering 
222 approach is that it can accommodate ambiguity in cluster assignments and provide more detailed 223 information about the structure of the dataset.

224

225

226

227 228

The objective of the fuzzy clustering algorithm is to minimize the within cluster variance and maximize between cluster variance; put another way, the objective is to minimize the distance between two objects belonging to the same cluster. This is accomplished through an iterative procedure where cluster membership is initiated and a coefficient of membership is calculated for each individual based on the distance of the individual to the centroid of each cluster. The process is repeated until new clustering iterations fail to maximize the objective. After clustering, a final coefficient of membership to each cluster is calculated for each individual. When an individual is assigned equal coefficients to all clusters, it is described as having 'complete fuzziness,' and can be imagined as falling in the 'middle ground' between all clusters; when an individual has a membership close to 1 to a particular cluster, the clustering is essentially hard (i.e., it is a partition). Dunn's normalized partition coefficient (1976) can be used to describe the overall fuzziness of an analysis, regardless of the number of clusters considered, where values close to 0 indicate high levels of fuzziness (near equal membership to all clusters), and values close to 1 indicate very low levels of fuzziness (i.e., hard partitions). After generating the coefficients of membership, one can find the hard partitioning scheme that most closely approximates the fuzzy clustering by assigning each individual to the cluster in which it has the largest membership.

One way to visualize the results of fuzzy clustering is by examining silhouette plots of the hard clusters. These plots are constructed of horizontal bars representing the silhouette coefficient (s(i) - a measure of that individual's similarity to other members of the same cluster) of each individual in the analysis, organized by hard cluster assignment. When s(i) is at its 
245 largest for an individual (close to 1), that means that the individual is much more similar to other

246 members of its cluster than it is to individuals outside of the cluster. When s(i) is low for an

247 individual (closer to 0), it means that the individual is equally similar to both members of its

248 cluster and members of other clusters. When an individual has an s(i) value that is negative, the

249 within cluster similarity is much smaller than the between cluster similarity. Finally, we can

250 calculate the mean silhouette coefficient (i.e., the mean silhouette coefficient of all samples in

251 the analysis) as a way of interpreting and validating the clustering. Kaufman and Rousseeuw

252 (2005) suggest that datasets with silhouette coefficients less than or equal to 0.25 have no

253 substantial structure, values between 0.26 and 0.50 indicate weak structure that could be artificial

254 and require additional methods to corroborate, values between 0.51 and 0.70 suggest reasonable

255 structure, and values between 0.71 and 1.0 suggest strong structure has been found.

256 Fuzzy clustering analyses were run using the function fanny in the R package cluster

257 (Maechler et al. 2016), and the same dissimilarity matrix for fuzzy clustering used for PCoA.

258 Fuzzy clustering requires the user to define the number of clusters $(\mathrm{k})$ to optimize. We chose to

259 examine clustering of $k=4,3$, and 2 clusters. We begin at four because this corresponds with the

260 number of named taxonomic entities focal to this study; three and two clusters were also

261 examined to explore the morphological signature of the data. We further examined the effect of

262 the membership exponent (a variable in the cluster optimization process) on our clustering

263 results. It has been shown that higher values (near two) lead to greater fuzziness while lower

264 values (near one) yield less fuzzy clustering (Kaufman and Rousseeuw 2005). We examined the

265 effect of this variable on clustering results by adjusting its value between 1.1 and 1.7, by

266 increments of 0.1 . We ran all fuzzy clustering analyses for 100,000 iterations, to assure

267 convergence. 
268 Estimating Position of Type Specimen in Morphospace. To explore the position of type

269 specimens in morphospace, we took the geographic position of each type specimen and found

270 the nearest population of the same species from which we took morphological measurements.

271 We make the assumption that these populations would have similar morphologies.

272

\section{Results}

274

Sampling. A total of 171 individuals were examined for this study. While normality is

275

276

277

278

279

280

281

282

283

284

variability in the data. The impact of outlier removal on downstream analyses was examined and found to have minimal influence (Fig. S1). After data cleaning and outlier removal, our final dataset consisted of Castilleja nana $(\mathrm{n}=29)$, C. pilosa var. longispica $(\mathrm{n}=23)$, C. pilosa var. pilosa $(\mathrm{n}=52)$, and C. pilosa var. steenensis $(\mathrm{n}=4)$, and covered the known ranges of each focal taxon (Fig. 2, open circles). Individuals measured, the herbarium housing each collection, and associated voucher information is available in the Supplemental Information, as well as raw data and associated analytical scripts. (Table S1, Dataset S1, Dataset S2, respectively)

Quantifying Morphological Variation. Violin dot plots of individual quantitative trait values, grouped by taxonomic identity revealed a great deal of overlap in raw trait values for each taxon across many traits. In some cases, this overlap occurs across all focal taxa, as in bract width and leaf width (Fig. 3), where all taxa have widely overlapping trait values. In other cases, the distribution of trait values distinguishes one of the focal taxa from the remaining three. For example, C. pilosa var. steenensis has a larger beak to tube ratio than the remaining taxa 
291 (meaning that the difference in length between the tube and the beak is greater), C. nana has a

292 longer bract than all varieties of $C$. pilosa and most $C$. pilosa var. longispica have shorter calyces

293 than other varieties of C. pilosa and C. nana. There are also cases of interspecific overlapping

294 trait distributions, as in plant height where C. nana and C. pilosa var. steenensis are generally

295 shorter in height than C. pilosa var. longispica and C. pilosa var pilosa. We see a similar pattern

296 of overlap in traits across taxa in our qualitative data. With the exception of the decumbent habit,

297 no one qualitative trait is found primarily in one taxon, let alone exclusively (Fig. 4). In general,

298 pubescence traits were equally variable across taxa, C. nana was the only taxon that occasionally

299 lacked lobes on the leaves, and C. pilosa var. pilosa and C. pilosa var. steenensis were the only

300 focal taxa that were never scored as having broader, deltoid shaped calyx lobes. Summary

301 statistics for raw values of continuous traits and raw counts of categorical traits can be found in

302 the supplement (Table S2 and S3, respectively).

303 Principal Coordinate Analysis. A Cailliez correction, equal to D' $=-0.5 *(\mathrm{D}+0.57237)$

$304 \wedge 2$, was applied to all negative eigenvalues. The position of each individual in the first two and

305 three principal coordinates are shown in Fig. 5, with 95\% confidence ellipses around the mean

306 position of each focal taxon in morphospace. An examination of axes 1 through 106 does not

307 change interpretation of the results presented here; the first two principal coordinate axes

308 represent the maximum morphological distance among individuals sampled, and the third axis

309 reveals no further distinction (Fig. 5).

310 In general, and considering all three principal coordinate axes, individuals identified as

311 Castilleja nana (yellow) occupy a different part of the scatterplot than those identified as $C$.

312 pilosa, including its named varieties (blue (var. pilosa), orange (var. longispica), and red (var.

313 steenensis)). Considering only those individuals identified as Castilleja pilosa, there is a large 
314 amount of overlap with no discernible position in morphospace unique to any variety (Fig. 5).

315 Confidence ellipses lend support to this conclusion and further suggests a greater distinction of

316 C. pilosa var. steenensis (in red) from any other focal taxon. The variation in distances of these

317 individuals lies along a different axis than the rest of the focal taxa; however, the effect of

318 sample size $(n=4)$ cannot be discounted.

319 Fuzzy Clustering. We performed seven fuzzy clustering analyses (corresponding to 320 seven different values of the membership exponent variable; values between 1.1 and 1.7, in

321 increments of 0.1$)$ for each of three possible numbers of clusters $(\mathrm{k}=4,3$, and 2$)$. Different values

322 of the membership exponent produced consistent results within each " $\mathrm{k}=\mathrm{X}$ number" of clusters.

323 For simplicity, we present the results from all clustering scenarios with a membership exponent 324 of 1.3 .

325 Fuzzy clustering analyses, regardless of number of clusters considered, resulted in

326 clusters with small silhouette coefficients (both within and across clusters), and low values for 327 the normalized Dunn coefficient (Fig. 6, Table 2). As cluster number was reduced, there 328 appeared to be some small improvement in these measures (average silhouette coefficient 329 increased from $0.2(\mathrm{k}=4)$ to $0.22(\mathrm{k}=3)$, and to $0.25(\mathrm{k}=2)$ and normalized Dunn coefficient 330 increased from $0.37(\mathrm{k}=4)$, to $0.38(\mathrm{k}=3)$, and $0.44(\mathrm{k}=2))$; however, overall these values are 331 extremely low. Generally speaking, regions of overlap in morphospace coincide with lower 332 probabilities of membership of each individual to each cluster (i.e., the probability of 333 membership to each cluster was higher, as opposed to having an overwhelming probability of 334 membership to any one single cluster), consistent across all clustering scenarios (Suppemental 335 Fig. 2). 
A somewhat subjective approach to quantifying the structure in a dataset is to calculate

337

338

339

340

341

342

343

344

345

346

347

348

349

350

351

352

353

354

355

356

357

the silhouette coefficient (SC) of the dataset (Kaufman and Rousseeuw 2005). This value is the maximum, average silhouette coefficient of all possible numbers of clusters, from $\mathrm{k}=2$ as a minimum, to $\mathrm{k}=n$ as a maximum $(n=108$, in this study). At $\mathrm{k}=53$, our standard 100,000 iterations of clustering were not enough to satisfy fuzzy clustering objectives, and we ran into convergence issues. However, considering $\mathrm{k}=2$ through $\mathrm{k}=53$ clusters, the average silhouette coefficients were highest at $\mathrm{k}=2$ (average $\mathrm{s}(\mathrm{i})=0.25)$, and steadily dropped as values of $\mathrm{k}$ increased.

To visualize the taxonomic composition of clusters, we painted the silhouettes with colors corresponding to the taxonomic identity of each individual. Across all three clustering schemes, one cluster is consistently composed of mostly $C$. nana individuals, with the remaining clusters being variously composed of all three varieties of $C$. pilosa. When we restrict the cluster number to two, the $C$. nana cluster begins to be more heavily composed of $C$. pilosa individuals (Fig. 6).

\section{Discussion}

Classifications are useful when they organize objects based on relationships, when they reflect similarities and differences among the constituent parts, and when they aid in the identification and placement of unknowns within the classification (Sokal 1974, de Queiroz and Donoghue 2011; de Queiroz and Donoghue 2013). The species description, based in part on the type specimen, plays an important role in the creation and implementation of classifications, but with a reliance on it comes the challenge of tracing and managing type collections and species descriptions through time - a problem that we are still dealing with (Hitchcock 1905; Dayrat 
359 2005). In addition, when objects are discrete and discontinuous, classifications are easy to build

360 and use; however, when there is continuous variation in characters used in the classification, this

361 becomes more difficult.

362 In this study we have closely examined morphology—a commonly used character for

363 describing taxonomic boundaries - for four named taxa, from across their ranges, in a species

364 complex known to be taxonomically difficult to diagnose. Here we have quantified a great deal

365 of overlap in raw character traits that are typically used to diagnose species (when using

366 taxonomic keys) in Castilleja (Fig. 3, 4). In some cases, these traits are continuous across

367 taxonomic boundaries (Fig. 3), emphasizing the extreme morphological similarity among these

368 named entities. We continue to see little distinction among current taxonomic groups when we

369 examine the morphospace described by all morphological characters that we measure here (Fig.

370 5). For example, C. pilosa, where we are essentially incapable of distinguishing taxonomic

371 varieties using morphology alone (Fig. 5), even in C. pilosa var. steenensis, considered the most

372 distinctive of the three varieties due to its isolation on Steens Mountain in SE Oregon (Hitchcock

373 et al. 1984). Finally, when we interrogate morphospace for evidence of structure, we find little

374 support (low silhouette widths for each cluster and low average silhouette widths for each

375 clustering scenario, Fig. 6) and equal assignment probabilities of individuals that occur in areas

376 of overlap to each cluster (Fig. S2).

377 And yet, despite the overall high levels of similarity we observe some consistent

378 distinction between individuals of C. nana and C. pilosa, indicating some morphological

379 distinction between taxa (Fig. 5). This is also supported by the results of fuzzy clustering

380 analyses that, regardless of the number of clusters considered, recover a cluster composed

381 primarily of $C$. nana, with C. pilosa individuals variously scattered among the remaining clusters 
382 (Fig. 6). Several continuous traits distinguish C. nana from C. pilosa (Fig.3; see also Fig. S3),

383 however, the overlapping tails of these distributions, and the nature of these distinguishing traits

384 (i.e., size and length traits that could be environmentally plastic), goes a long way towards

385 explaining the morphological confusion that has plagued this complex historically.

386 It is clear that geographic and ecological characters must have played a dominant role in

387 shaping the species descriptions in this complex. This is apparent from the species descriptions

388 included both in regional and genus-wide treatments (Cronquist et al. 1984; Hitchcock et al.

389 1984; Wetherwax et al. 2012), as well as the inferred species ranges (Fig. 2). For example, $C$.

390 nana does not occur in the northern limits of the C. pilosa range. So, if you encounter a relatively

391 small individual in Idaho, there is no way to confuse it with C. nana (a California and Nevada

392 species), as the ranges do not overlap, and the regional treatment does not consider C. nana

393 (Hitchcock et al. 1984). Similarly, C. pilosa var. steenensis only occurs on Steens Mountain in

394 Eastern Oregon. If you found a relatively small individual in central Oregon, you could only

395 classify it as C. pilosa var. pilosa, using these regional treatments.

396 When species occur sympatrically, however, the distinction between named entities

397 becomes much more difficult to parse. In the Sierra Nevada, C. pilosa var. pilosa (a moderate

398 elevation taxon) and C. nana (a high elevation taxon) can co-occur at the limits of their

399 elevational ranges (high and low, respectively) where environments are heterogeneous.

400 Similarly, C. pilosa var. pilosa and C. pilosa var. steenensis can co-occur on the western slopes

401 of Steens Mountain in the transition area between the high, exposed ridge and the surrounding

402 lower elevation steppe. In heterogeneous habitats and at ecological boundaries, phenotypes can

403 be accentuated and variable (Van Kleunen and Fischer 2005), potentially in response to local

404 microhabitat conditions such as light availability and precipitation (Schlichting 1986; Dorn et al. 
405 2000; van Kleunen et al. 2000; Nicotra et al. 2010). As a result, it is possible that in these areas

406 of sympatry that correspond with environmental transitions, individuals could experience

407 extreme conditions that may affect the morphological traits that we examine when we try to

408 identify unknowns. We see this in several individuals from the Sierra Nevada that have extreme

409 values in the traits that distinguish C. nana and C. pilosa (Fig. 7). Furthermore, these are the

410 individuals that occur in the region of overlap in morphospace between these two taxa (Fig. 7).

411 In some cases, these regions of sympatry also correspond with hotspots of taxonomic

412 synonymy historically - i.e., these sympatric areas are places where synonyms of currently

413 accepted taxa were described (Fig. 8). For example, the area surrounding Lake Tahoe has seen

414 the description of four distinct taxa (Castilleja jusselii Eastw. (Eastwood 1940), Orthocarpus

415 pilosus S. Wats. (Watson 1871), Castilleja inconspicua A.Nelson \& P.B.Kenn (Nelson and

416 Kennedy 1906), Castilleja nana Eastw. (Eastwood 1902)), two of which (O. pilosus and C.

417 nana) are the type specimens for Castilleja nana and Castilleja pilosa (Fig. 8). The remaining

418 two taxa were later incorporated into C. nana (C. inconspicua) and C. pilosa (C. jusselii),

419 effectively meaning that these entities are no different from C. nana and C. pilosa. However,

420 when we place our best approximation of Castilleja inconspicua in morphospace (i.e., a

421 specimen of the same taxon (C. inconspicua is a synonym of $C$. nana) that was measured by us

422 that is as geographically close to the type collection of $C$. inconspicua as possible), we find that

423 this collection occupies a region of morphospace very different from that of the type collection

424 of $C$. nana (Fig. 8). By including this species into the concept of C. nana through

425 synonymization in the Intermountain Flora (Cronquist et al. 1984), the amount of variation

426 attributed to $C$. nana likely expanded. 
Areas of sympatry are not the only source of potential confusion in the taxonomic history

428

429

430

431

432

433

434

435

436

437

438

439

440

\section{Conclusion}

442

443

444

445

446

447

448

449

\section{Conclusion}

of either taxon. For example, the synonymization of Castilleja lapidicola A.Heller (Heller 1912)

in eastern Nevada with $C$. nana also expanded the region of morphospace attributed to C. nana

((Cronquist et al. 1984), Fig. 8). Similarly, in northern California the inclusion of C. ochracea

Eastw. (Eastwood 1941) and C. pisttacinus (Orthocarpus psittacinus Eastw. (Eastwood 1902);

C. psittacina (Eastw.) Pennell (Abrams 1951)) increased the area of morphospace occupied by $C$.

pilosa ((Cronquist et al. 1984); Fig. 8). Ultimately, the qualitative decisions made about species

boundaries based on regional treatments has extended and inflated the morphological concepts of both taxa. By going through this procedure of quantifying morphological variation, we can

visualize what morphological variation the taxonomy currently embodies. It is apparent that the morphological concept of both $C$. nana and C. pilosa have expanded through the incorporation of additional taxa as synonyms, and it is possible that the species description of both taxa may no longer represent the features of either taxon.

The inflation of morphological variation attributed to C. nana and C. pilosa during species level revisions, much of them regionally based, in addition to an apparent reliance on potentially plastic morphological characters to distinguish species in sympatry, has resulted in a great deal of morphological confusion in this complex. This likely contributes to the tumultuous taxonomic history of these taxa, and suggests that relying on morphology alone to define species boundaries in this complex is problematic. This is where molecular and ecological lines of evidence will be incredibly important to delimit species. In a robust and integrated delimitation of species, we may find that taxa that have been synonymized are not truly part of their 
450 corresponding taxa, or vice versa. Subsequent classifications should reflect these boundaries and 451 highlight the similarities and differences between them.

452 Here we have begun that process by quantifying morphological variation in this species 453 complex and we have estimated the position of type specimens in that space. The next steps in 454 this group will be to gather molecular and ecological evidence to contribute to a robust species 455 delimitation that is based on multiple lines of evidence. With all data in hand, we can more 456 confidently apply names, whether that is applying an old name, a new name, or combining them 457 all in one.

\section{Acknowledgements}

459 The authors would like to thank the following herbaria for providing access to specimens for 460 morphological measurements: College of Idaho Harold M. Tucker Herbarium (CIC), University 461 of Idaho Stillinger Herbarium (ID), University of Montana Herbarium (MONTU), Oregon State 462 University Herbarium (OSC), University of Wyoming Rocky Mountain Herbarium (RM), 463 Washington State University Marion Ownbey Herbarium (WS), University of Washington 464 Herbarium (WTU). Additionally, the authors would like to thank two anonymous reviewers 465 whose thoughtful comments and suggestions were greatly appreciated and contributed to the 466 improvement of the manuscript. 


\section{References}

469 Abrams, L. 1951. Castilleja. Illustrated Flora of the Pacific States. Volume 3, p. 851.

$470 \quad$ Stanford University Press.

471 Barley, A. J., J. White, A. C. Diesmos, and R. M. Brown. 2013. The challenge of species

472 delimitation at the extremes: diversification without morphological change in philippine

473 sun skinks. Evolution. 67:3556-3572.

474 Baum, D. A., and K. L. Shaw. 1995. Genealogical perspectives on the species problem.

475 Experimental and molecular approaches to plant biosystematics. 53:289-303.

476 Cailliez, F. 1983. The analytical solution of the additive constant problem.

477 Psychometrika. 48:305-308.

478 Carstens, B. C., T. A. Pelletier, N. M. Reid, and J. D. Satler. 2013. How to fail at species 479 delimitation. Molecular Ecology. 22:4369-4383.

480 Chuang, T. I., and L. R. Heckard. 1991. Generic realignment and synopsis of subtribe 481 Castillejinae (Scrophulariaceae-tribe Pediculareae). Systematic Botany. 644-666.

482 Cronquist, A. J., A. H. Holmgren, N. H. Holmgren, and J. L. Reveal. 1984. Castilleja. Pp.

483 476-496 in Vascular Plants of the Intermountain West, U.S.A. eds. A. J. Cronquist,

484 A. H. Holmgren, N. H. Holmgren, J. L. Reveal, \& P. K. Holmgren. Hafner

485 Pub. Co., New York.

486 Dayrat, B. 2005. Towards integrative taxonomy. Biological Journal of the Linnean

$487 \quad$ Society. 85:407-415.

488 de Queiroz, K. 2007. Species concepts and species delimitation. Systematic Biology.

$489 \quad 56: 879-886$.

490 de Queiroz, K. 2009. The General Lineage Concept of Species and the Defining 
491

492

493

494

495

496

497

498

499

500

501

502

503

504

505

506

507

508

509

510

511

512

513

Properties of the Species Category. Pp. 49-89. in Species: New interdisciplinary essays. ed. R.A. Wilson. Cambridge, MIT Press.

de Queiroz, K. and M. Donoghue. 2011. Phylogenetic Nomenclature, Three-Taxon Statements, and Unnecessary Name Changes. Systematic Biology. 60 : 887-892.

de Queiroz, K. and M. Donoghue. 2013. Phylogenetic Nomenclature, Hierarchical Information, and Testability. Systematic Biology. 62: 167-174.

Dejaco, T., M. Gassner, W. Arthofer, B. C. Schlick-Steiner, and F. M. Steiner. 2016. Taxonomist's Nightmare ... Evolutionist's Delight : An Integrative Approach Resolves Species Limits in Jumping Bristletails Despite Widespread Hybridization and Parthenogenesis. Systematic Biology. 65:947-974.

Dorn, L. A., E. H. Pyle, and J. Schmitt. 2000. Plasticity to light cues and resources in Arabidopsis thaliana: Testing for adaptive value and costs. Evolution. 54:19821994.

Dunn, J. C. 1976. Indices of partition fuzziness and the detection of clusters in large data sets. in Fuzzy Automata and Decision Processes. ed. M. Gupta. New York: Elsevier.

Eastwood, A. 1902. Castilleja nana. Proceedings of the California Academy of Sciences, Series 3. 2:289-290.

Eastwood, A. 1902. Orthocarpus psittacinus. Bulletin of the Torrey Botanical Club. 29:78.

Eastwood, A. 1940. Castilleja jussellii. Leaflets of Western Botany. 2:243-244.

Eastwood, A. 1941. Castilleja ochracea. Leaflets of Western Botany. 3:91.

Ence, D. D., and B. C. Carstens. 2010. SpedeSTEM: a rapid and accurate method for 
$514 \quad$ species delimitation. Molecular Ecology Resources. 11:473-480.

515 Freudenstein, J. V., M. B. Broe, R. A. Folk, and B. T. Sinn. 2016. Biodiversity and the 516 Species Concept - Lineages are not Enough. Systematic Biology. 66:644-656.

517 Giarla, T. C., R. S. Voss, and S. A. Jansa. 2014. Molecular Phylogenetics and Evolution. $518 \quad 70: 137-151$.

519 Gower, J. C. 1966. Some Distance Properties of Latent Root and Vector Methods Used in 520 Multivariate Analysis. Biometrika. 53:325-\&.

521 Gower, J. C. 1971. A General Coefficient of Similarity and Some of Its Properties. $522 \quad$ Biometrics. 27:857-871.

523 Gower, J. C. 1982. Euclidean distance geometry. Math. Scientist. 7:1-14.

524 Heckard, L. R., and T. I. Chuang. 1977. Chromosome-Numbers, Polyploidy, and 525 Hybridization in Castilleja (Scrophulariaceae) of Great Basin and Rocky $526 \quad$ Mountains. Brittonia. 29:159-172.

527 Heller, A. 1912. Castilleja lapidicola. Muhlenbergia; a journal of botany. 8:49-50.

528 Hersch-Green, E. I., and R. Cronn. 2009. Tangled trios?: Characterizing a hybrid zone in 529 Castilleja (Orobanchaceae). American Journal of Botany. 96:1519-1531.

530 Hitchcock, A. S. 1905. Nomenclatorial type specimens of plant species. Science. $531 \quad 21: 0828-0832$.

532 Hitchcock, C. H., A. J. Cronquist, F. M. Ownbey, and J. W. Thompson. 1984. Castilleja. 533 Pp. 295-326 in Vascular Plants of the Pacific Northwest. Seattle: University of $534 \quad$ Washington Press.

535 Kaufman, L., and P. J. Rousseeuw. 2005. Finding Groups in Data. John Wiley \& Sons, $536 \quad$ Inc. 
537 Legendre, P., and L. Legendre. 1998. Numerical ecology: second English edition.

$538 \quad$ Elsevier.

539 Maechler, M., P. Rousseeuw, A. Struyf, M. Hubert, and K. Hornik. 2016. cluster: Cluster

$540 \quad$ Analysis Basics and Extensions.

541 Nelson, A. and P. B. Kennedy. 1906. Castilleja inconspicua. Proceedings of the

$542 \quad$ Biological Society of Washington. 19: 38.

543 Nicotra, A. B., O. K. Atkin, S. P. Bonser, A. M. Davidson, E. J. Finnegan, U. Mathesius,

544 P. Poot, M.D. Purugganan, C.L. Richards, F. Valladares, and M. van Kleunen. 2010. Plant

545 phenotypic plasticity in a changing climate. Trends in

$546 \quad$ plant science. 15:684-692.

547 Padial, J. M., A. Miralles, I. De la Riva, and M. Vences. 2010. The integrative future of $548 \quad$ taxonomy. Frontiers in Zoology. 7:16.

549 Paradis, E., J. Claude, and K. Strimmer. 2004. APE: analyses of phylogenetics and $550 \quad$ evolution in R language. Bioinformatics. 20:289-290.

551 Pohl, R. W. 1965. Dissecting equipment and materials for the study of minute plant $552 \quad$ structures. Rhodora. 67:95-96.

553 R Core Team. 2017. R: A language and environment for statistical computing. R

554 Foundation for Statistical Computing, Vienna, Austria. Available from:

$555 \quad$ https://www.R-project.org/.

556 Schlichting, C. D. 1986. The Evolution of Phenotypic Plasticity in Plants. Annual Review

557 of Ecology and Systematics. 17:667-693.

558 Schlick-Steiner, B. C., F. M. Steiner, B. Seifert, C. Stauffer, E. Christian, and R. H.

559 Crozier. 2010. Integrative Taxonomy: A Multisource Approach to Exploring 
Biodiversity. Annual Review of Entomology. 55:421-438.

561 Sneath, P. H. A., and R. R. Sokal. 1973. Numerical Taxonomy: The Principles and $562 \quad$ Practice of Numerical Classification.

563 Sokal, R. R. 1974. Classification - Purposes, Principles, Progress, Prospects. Science. $564 \quad 185: 1115-1123$.

565 Tank, D. C., and R. G. Olmstead. 2008. From annuals to perennials: phylogeny of 566 subtribe Castillejinae (Orobanchaceae). American Journal of Botany. 95:608-625.

567 Tank, D. C., J. M. Egger, and R. G. Olmstead. 2009. Phylogenetic Classification of 568 Subtribe Castillejinae (Orobanchaceae). Systematic Botany. 34:182-197.

569 Van Kleunen, M., and M. Fischer. 2005. Constraints on the evolution of adaptive 570 phenotypic plasticity in plants. New Phytologist. 166:49-60.

571 van Kleunen, M., M. Fischer, and B. Schmid. 2000. Clonal integration in Ranunculus 572 reptans: by-product or adaptation? Journal of Evolutionary Biology. 13:237-248.

573 Van Valen, L. 1976. Ecological Species, Multispecies, and Oaks. Taxon. 25:233-239.

574 Watson, S. 1871. Orthocarpus pilosus. United States Geological Exploration of the $575 \quad$ Fortieth Parallel. Vol. 5, Botany.

576 Wetherwax, M., T. I. Chuang, and L. R. Heckard. 2012. Castilleja. Jepson Flora Project. 577 Available from: http://www.ucjeps.berkeley.edu/eflora. 


\section{Figure 1}

Schematic representing the amount of variation attributed to a species through time.

Species "A" and "B" are described, anchored by type collections "A" and "B", and including variation around the type (smaller, dark continuous lines). Species descriptions of "A" and "B" are updated in regional treatments and floristic studies to incorporate variation observed in the field (arrows and lighter, continuous lines). Revisionary work recognizes overlap in variation in species "A" and "B" and synonymizes species "B" with species "A" (dark, most inclusive continuous line). 


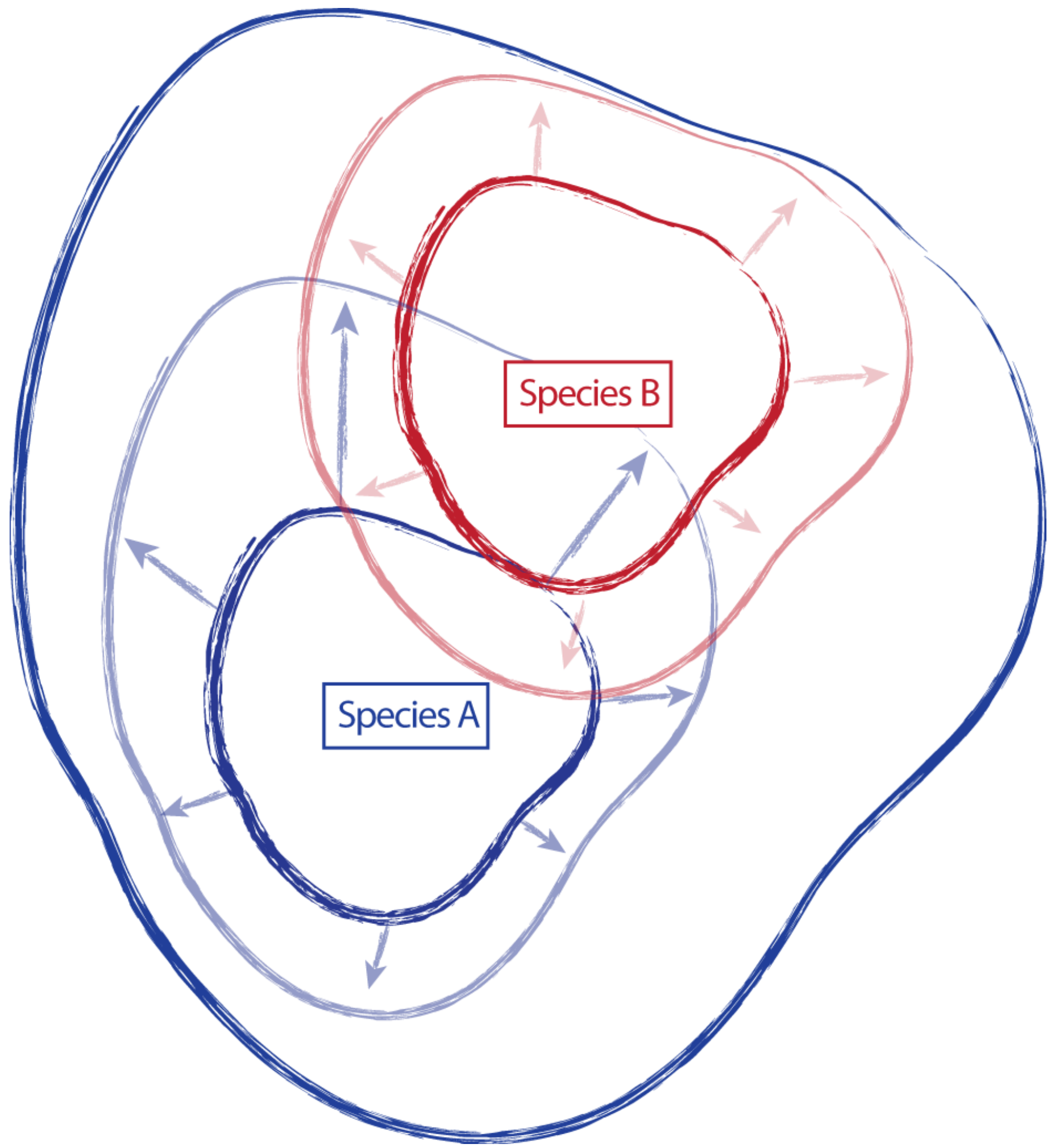




\section{Figure 2}

Distribution of focal taxa and diagrams of species morphology.

Distribution of focal taxa (A) and diagrams of species morphology (B). Filled circles represent specimens used to estimate ranges only (accessed through regional databases Consortium of Pacific Northwest Herbaria (pnwherbaria.org); Southwest Environmental Information Network (SEINet; swbiodiversity.org); University and Jepson Herbaria Specimen Portal (webapps.cspace.berkeley.edu); New York Botanical Garden (NYBG; nybg.org); Rocky Mountain Herbarium (RM; rmh.uwyo.edu)); open circles are individuals measured in this study. Castilleja pilosa var. pilosa (blue), C. pilosa var. longispica (orange), C. pilosa var. steenensis (red), C. nana (yellow). Photo Credit: JM Egger. Map data @2017 Google.
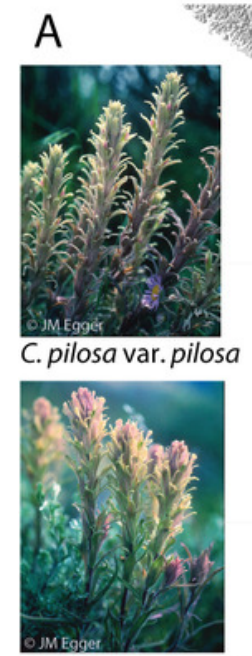

C. pilosa var. longispica

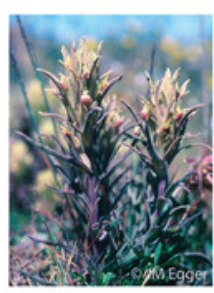

C. pilosa var. steenensis

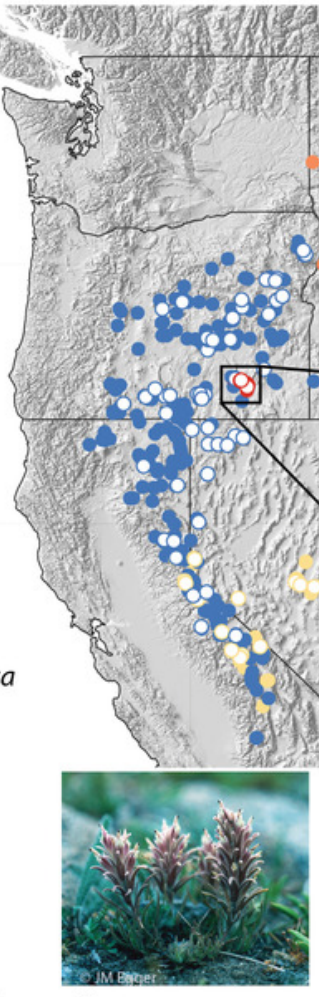

C. nana
B

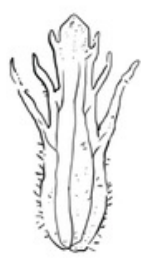

Bract
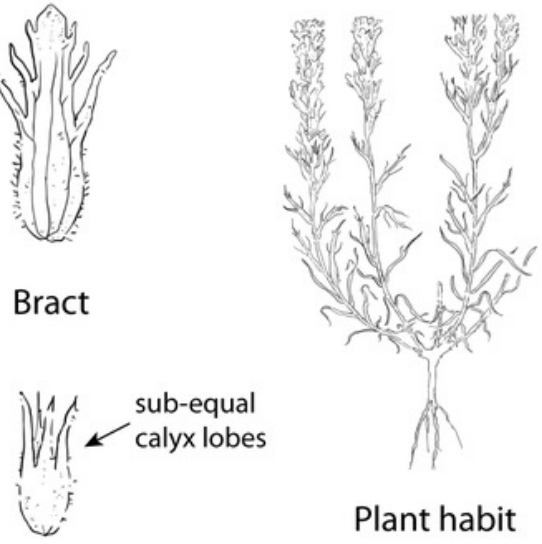

Plant habit

Calyx

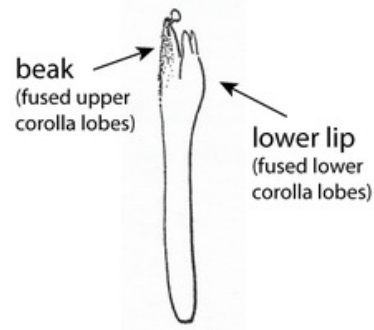

Corolla 


\section{Figure 3}

Raw trait values for continuous traits.

Violin dot plots of raw trait values (A - K) for the continuous traits measured in this study. $C$. pilosa var. longispica (orange), C. pilosa var. pilosa (blue), C. pilosa var. steenensis (red), and C. nana (yellow).
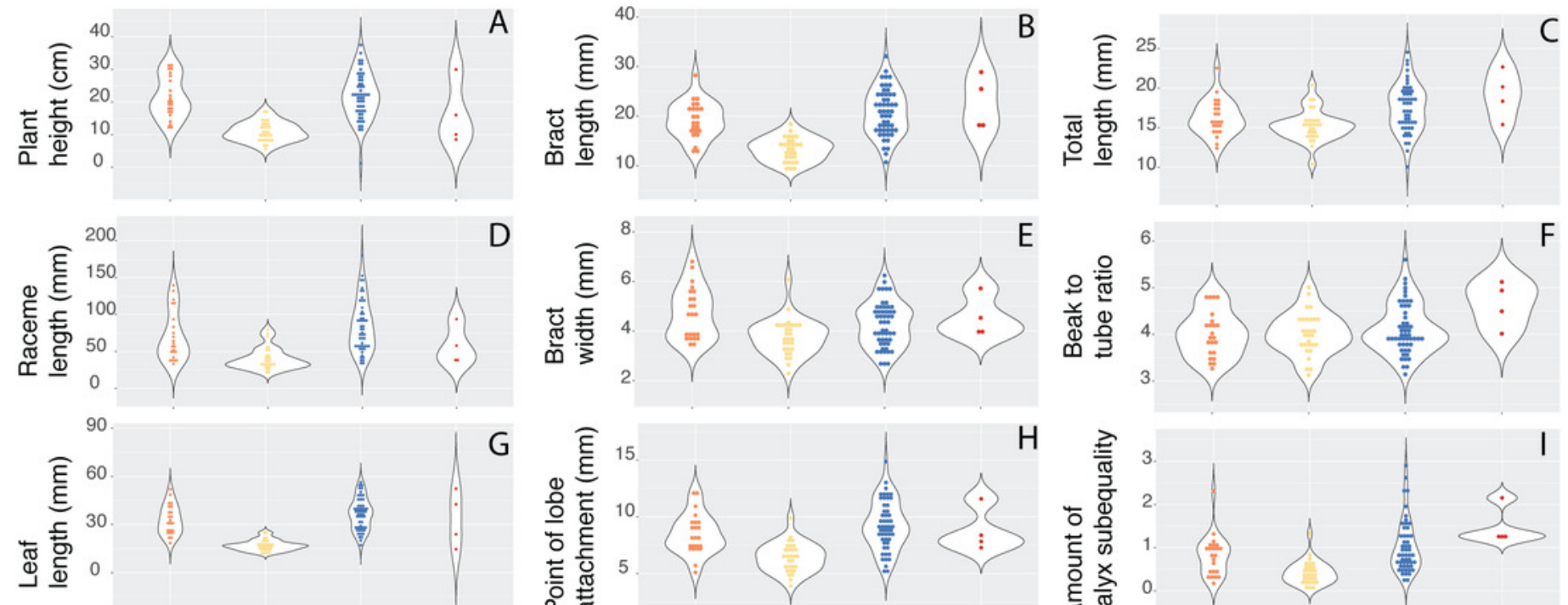

$\mathrm{G}$
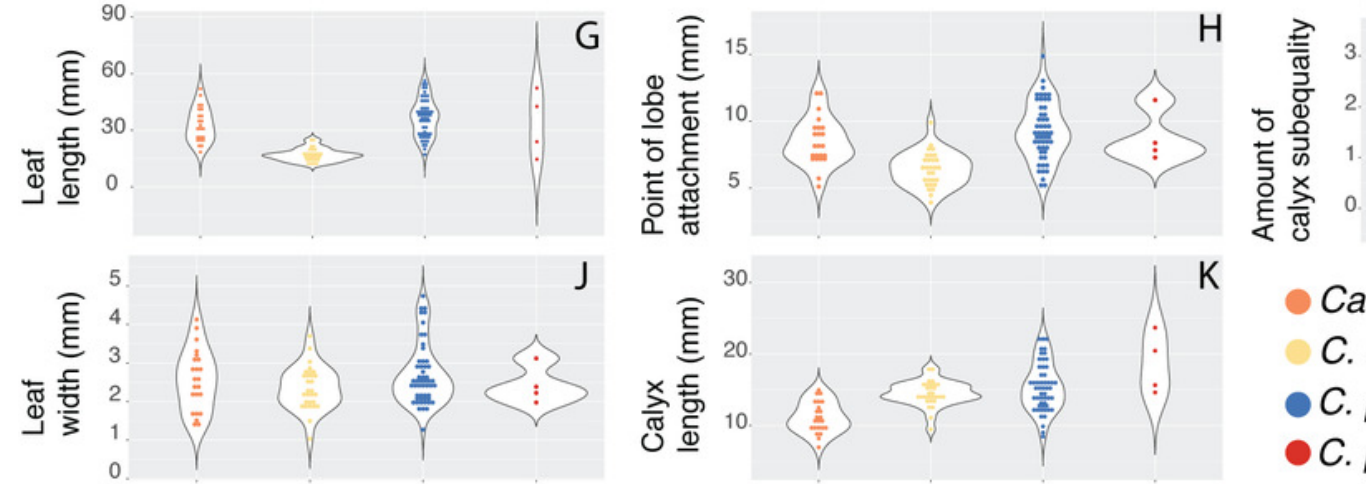

$\mathrm{K}$

- Castilleja pilosa var. longispica

C. nana

- C. pilosa var. pilosa

- C. pilosa var. steenensis 


\section{Figure 4}

Summary of counts for categorical characters.

Summary of counts for categorical characters (A - F) measured here. Columns represent focal taxa whose area represents all individuals identified to that taxon in our dataset. Shading represents different character states scored for each individual. Dashes represent a character state unobserved in a particular taxon. For calyx lobe shapes, numbers are used in place of trait descriptions for simplicity. These correspond to: 1) linear, 2) lanceolate/linear, 3) lanceolate, 4) deltoid/lanceolate, and 5) deltoid. 
A Glandular hairs

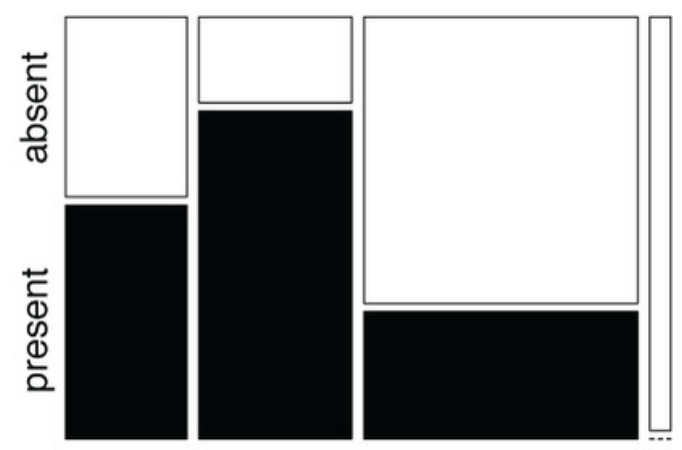

C Recurved hairs

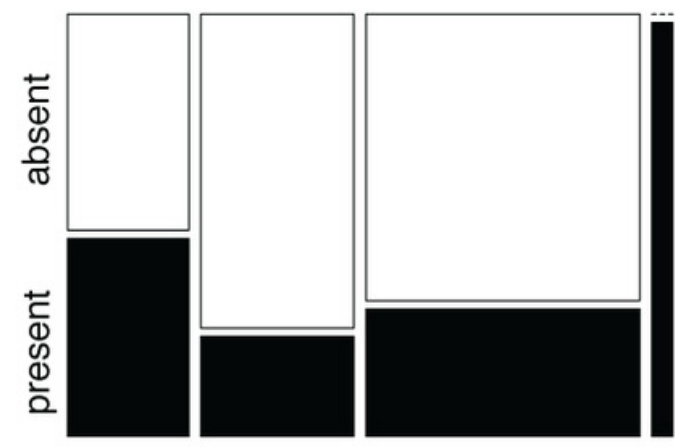

$\mathrm{E}$

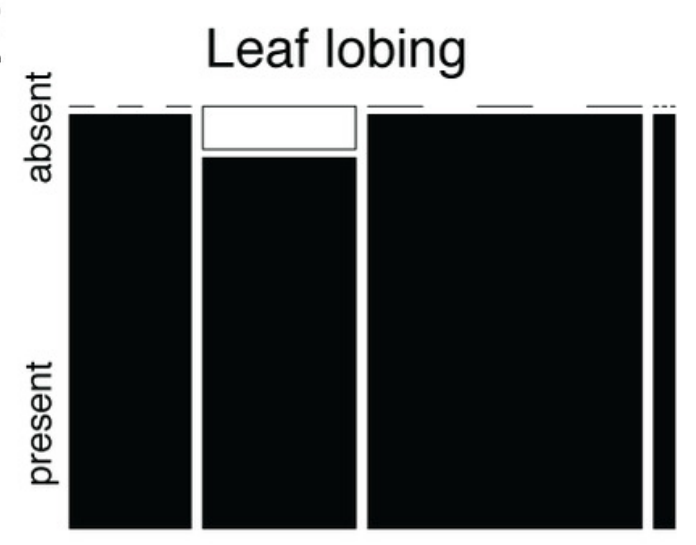

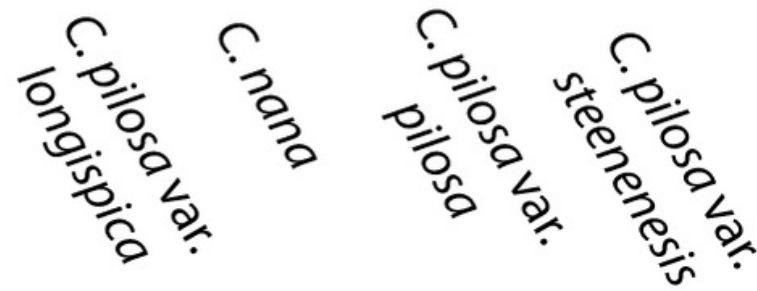

Length of herbage

B pubescence

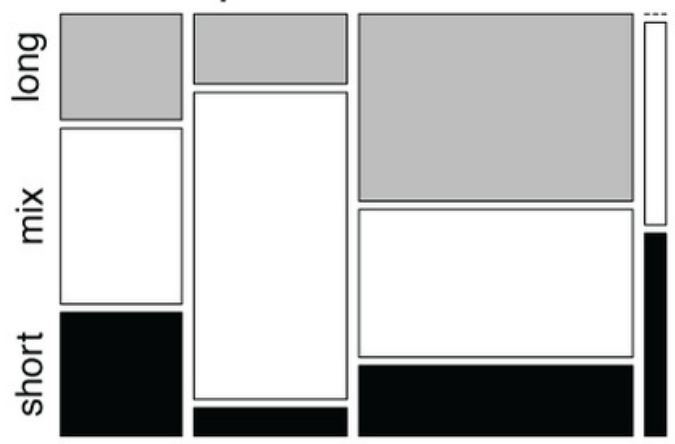

D Decumbent habit

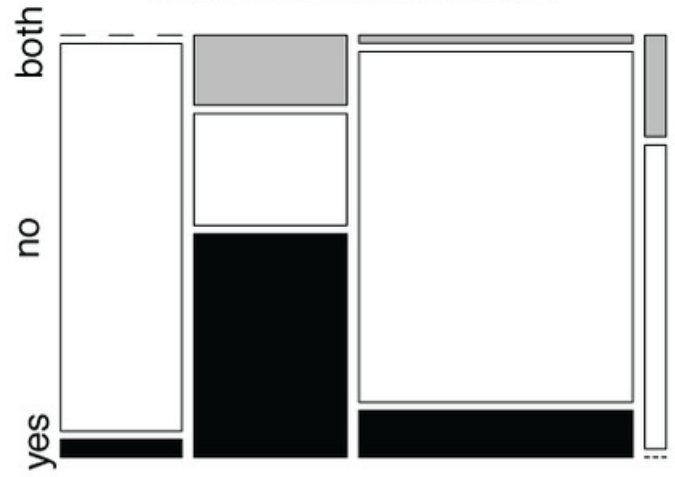

Calyx lobe shape

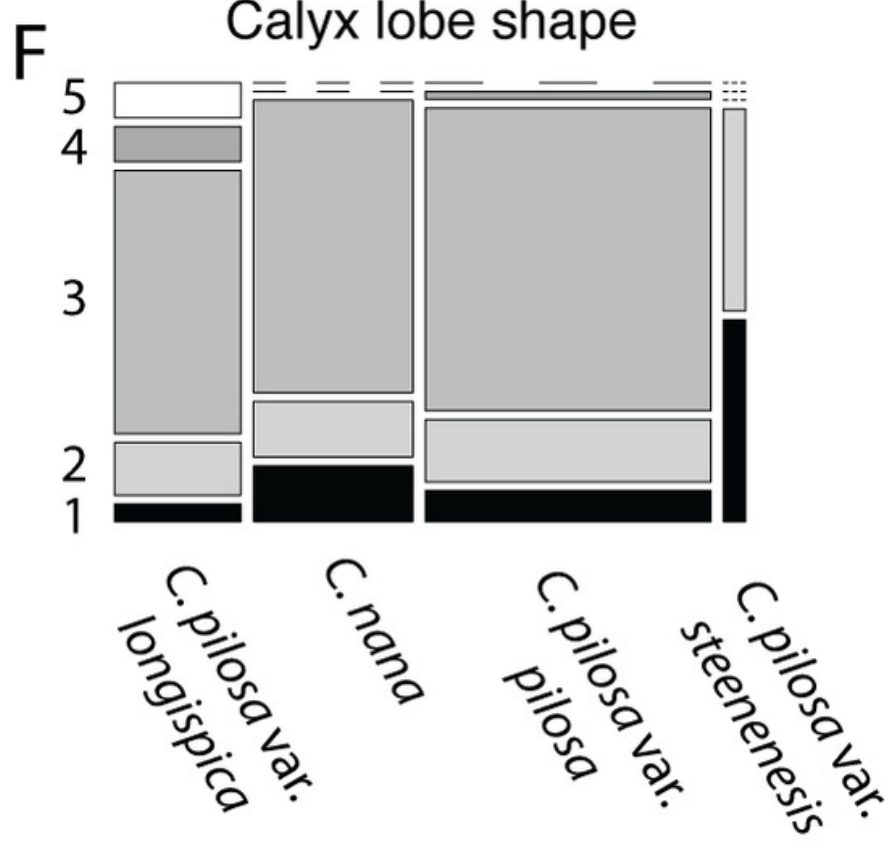


Figure 5

Results of Principle Coordinate Analysis.

Results of Principle Coordinate Analysis (PCOA) considering the first two axes of variation (A) and including a third axis (B). Individuals are represented by points in morphospace, and colored according to species identification: Castilleja pilosa var. pilosa (blue), C. pilosa var. longispica (orange), C. pilosa var. steenensis (red), and C. nana (yellow).

A

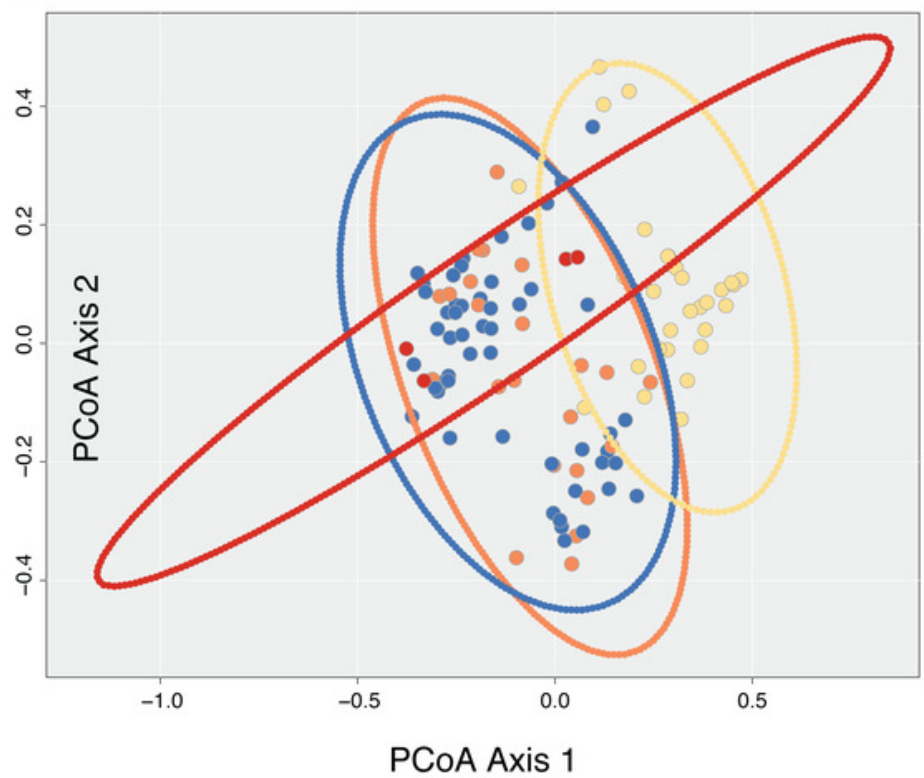

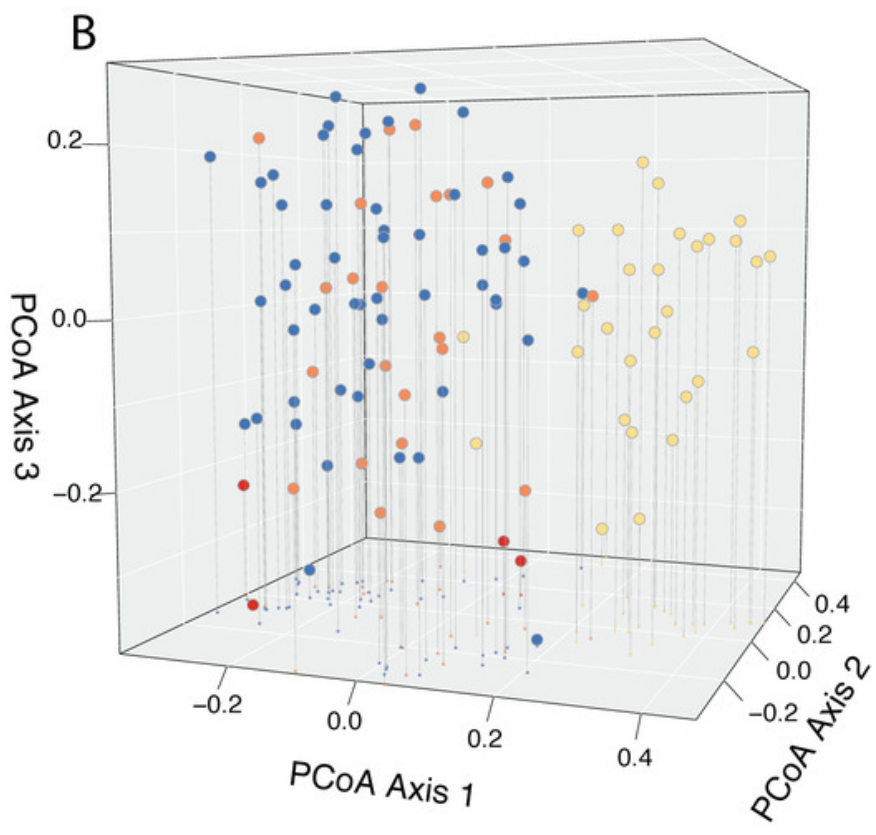




\section{Figure 6}

Results of fuzzy clustering.

Results of fuzzy clustering for $k=4$ clusters (A), $k=3$ clusters (B), and $k=2$ clusters (C). For each set of silhouettes, the width of each bar corresponds to the silhouette coefficient for that individual in the analysis. The silhouette width is a measure of that individual's similarity to other members of the same cluster-when large (close to 1), that means that the individual is much more similar to other members of its cluster than it is to individuals outside of the cluster; when low (closer to 0 ), it means that the individual is equally similar to both members of its cluster and members of other clusters; when negative, the within cluster similarity is much smaller than the between cluster similarity. We also report the average silhouette coefficient for each analysis $(k=4,3,2)$. Bars are painted with colors corresponding to species identification and numbered for cross-referencing against Supplemental Table 4: Castilleja pilosa var. pilosa (blue), C. pilosa var. longispica (orange), C. pilosa var. steenensis (red), and C. nana (yellow). 


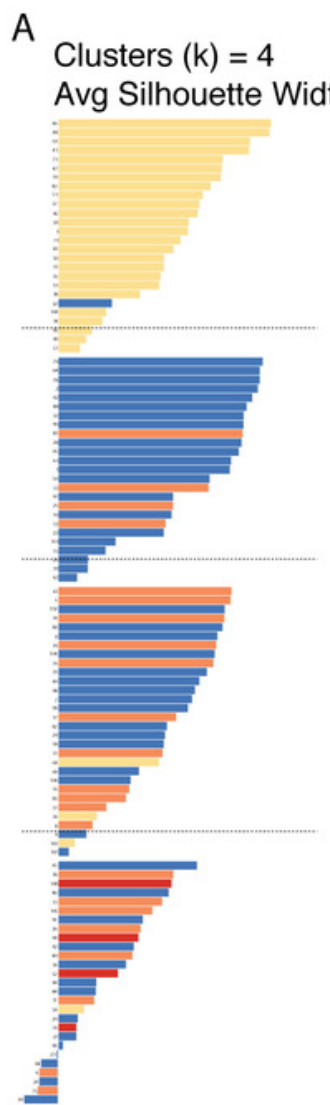

B Clusters $(k)=3$
Avg Silhouette Width $=0.22$

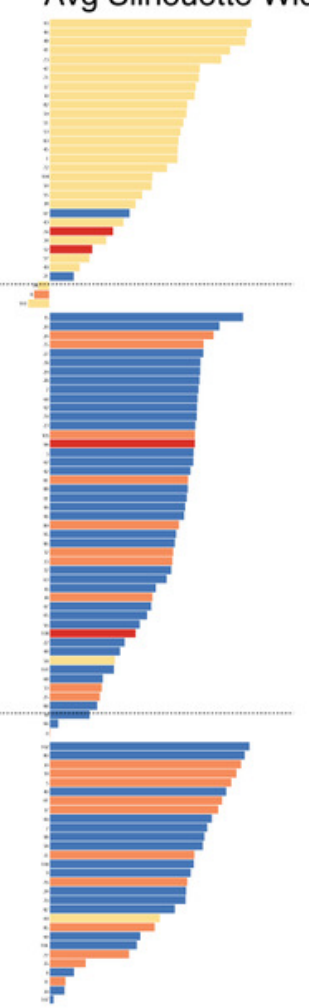

C Clusters $(k)=2$ Avg Silhouette Width $=0.25$

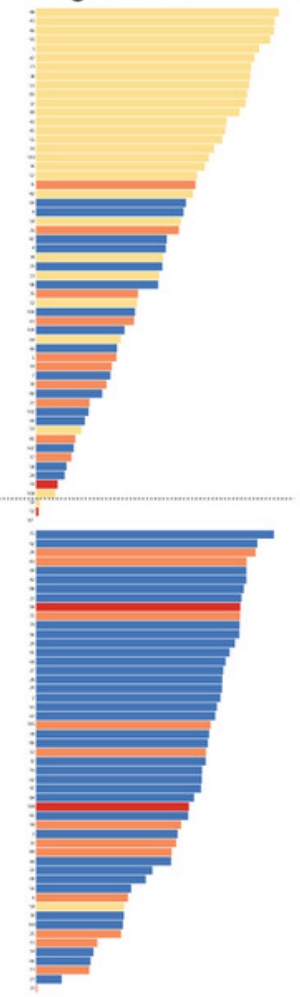

$\begin{array}{llllll}0.0 & 0.2 & 0.4 & 0.6 & 0.8 & 1.0\end{array}$

\begin{tabular}{lllll}
\hline 0.0 & 0.2 & 0.4 & 0.6 & 0.8
\end{tabular}

$\begin{array}{lll}0 & 0.0 & 0.2\end{array}$

Castilleja pilosa var. longispica

C. nana

¿C. pilosa var. pilosa

C. pilosa var. steenensis 
Figure 7

Position of individuals in morphospace and geographic space.

Position of individuals with extreme trait values in morphospace (A) and in geographic space (B). Inividuals are color-coded according to taxonomic identification: Castilleja pilosa var. pilosa (blue), C. pilosa var. longispica (orange), C. pilosa var. steenensis (red), and C. nana (yellow). Histograms at the top of the diagram show trait distributions for $C$. nana (yellow) and C. pilosa (including all varieties, blue) for simplification. Vertical lines represent raw trait values and are color-coded corresponding to taxonomic identification.
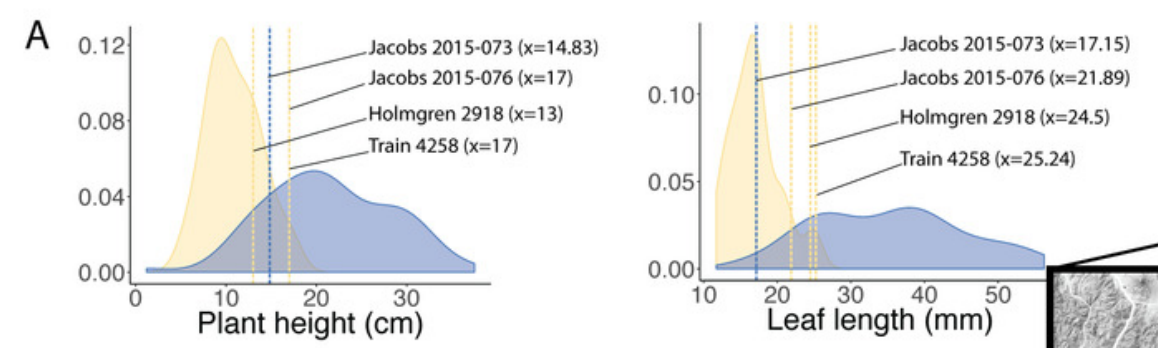

C

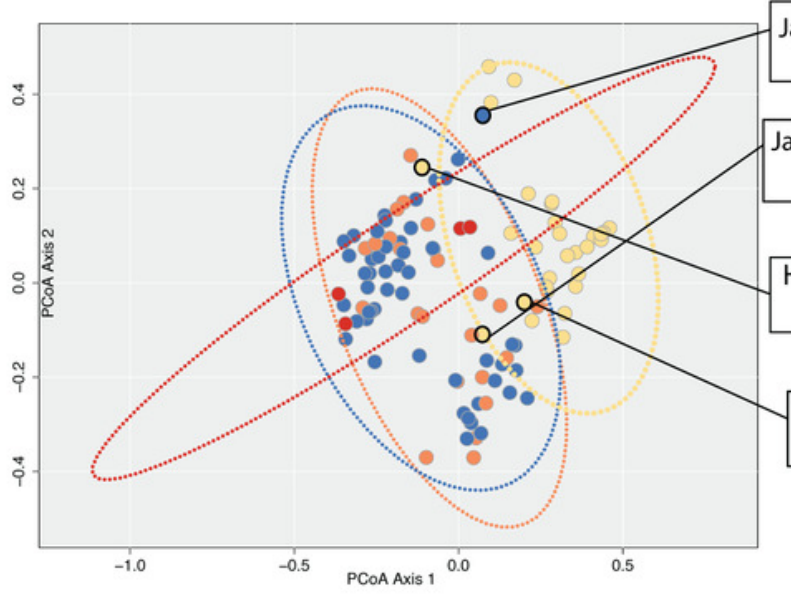

Jacobs 2015-073 (C. pilosa) Jacobs 2015-076 (C. nana)

Holmgren 2918 (C. nana)

Train 4258 (C. nana) 


\section{Figure 8}

Position of type collections.

Position of type collections of focal taxa and associated synonyms, within the known ranges of each taxon (A) and the corresponding position of the nearest geographic individual that we have measurements for in our dataset is identified in morphospace (B). Inividuals are colorcoded according to taxonomic identification: Castilleja pilosa var. pilosa (blue), C. pilosa var. longispica (orange), C. pilosa var. steenensis (red), and C. nana (yellow). 


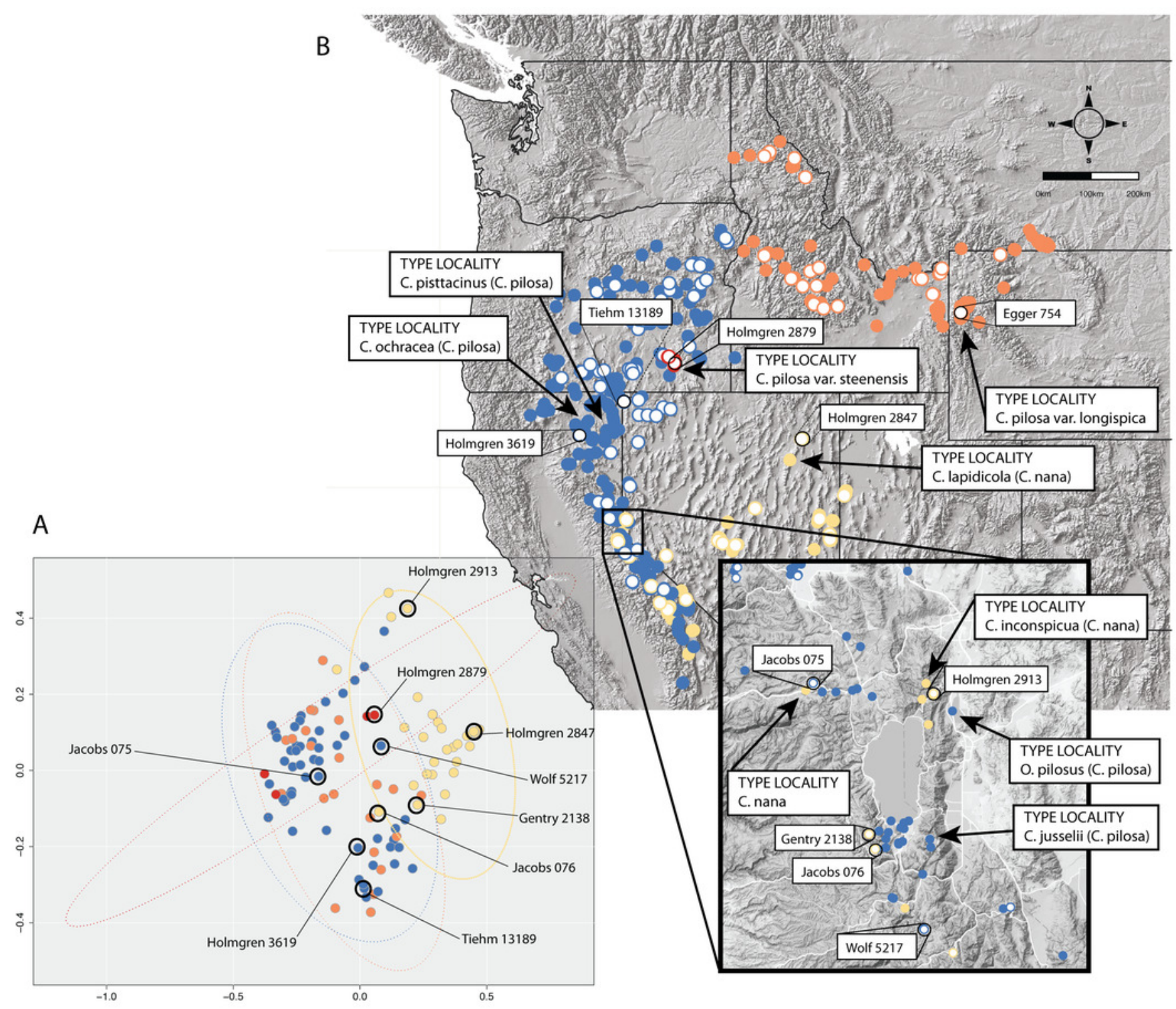




\section{Table $\mathbf{1}$ (on next page)}

Morphological characters measured in Castilleja.

Morphological characters measured in Castilleja. The first column following the character name reflects the type of character measured: continuous (C), nominal (N), or dichotomous (D); the second column provides the unit of measurement, the number of levels for nominal or ordinal data, and (when necessary) the formula for character calculation. Asterisks (*) indicate characters were not directly included in analyses, but used to calculate composite variables. 


\begin{tabular}{|c|c|c|c|}
\hline & Character & $\begin{array}{l}\text { Data } \\
\text { type }\end{array}$ & $\begin{array}{l}\text { Unit / level of } \\
\text { measure }\end{array}$ \\
\hline \multicolumn{4}{|l|}{ Habit } \\
\hline 1 & Plant height & $\mathrm{C}$ & $\mathrm{cm}$ \\
\hline 2 & Decumbent at base & $\mathrm{N}$ & 3 \\
\hline \multicolumn{4}{|l|}{$\begin{array}{l}\text { Surface } \\
\text { Textures }\end{array}$} \\
\hline 3 & Length of herbage pubescence & $\mathrm{N}$ & 3 \\
\hline 4 & Recurved hairs present & $\mathrm{D}$ & 2 \\
\hline 5 & Glandular hairs present & $\mathrm{D}$ & 2 \\
\hline \multicolumn{4}{|l|}{ Inflorescence } \\
\hline 6 & Number of racemes per stem & $\mathrm{C}$ & 8 \\
\hline 7 & Length of raceme & $\mathrm{C}$ & $\mathrm{mm}$ \\
\hline \multicolumn{4}{|l|}{ Leaf } \\
\hline 8 & Length of leaf & $\mathrm{C}$ & $\mathrm{mm}$ \\
\hline 9 & Width of leaf & $\mathrm{C}$ & $\mathrm{mm}$ \\
\hline 10 & Leaf lobing & $\mathrm{D}$ & 2 \\
\hline \multicolumn{4}{|l|}{ Bract } \\
\hline 11 & Length of bract & $\mathrm{C}$ & $\mathrm{mm}$ \\
\hline 12 & Width of bract & $\mathrm{C}$ & $\mathrm{mm}$ \\
\hline 13 & Number of secondary lobe pairs & $\mathrm{C}$ & 4 \\
\hline 14 & Point of lobe attachment & $\mathrm{C}$ & $\mathrm{mm}$ \\
\hline \multicolumn{4}{|l|}{ Calyx } \\
\hline 15 & Length of calyx & $\mathrm{C}$ & $\mathrm{mm}$ \\
\hline 16 & Tip of calyx to sinus 1 & $\mathrm{C}$ & $\mathrm{mm}^{*}$ \\
\hline 17 & Tip of calyx to sinus 2 & $\mathrm{C}$ & $\mathrm{mm}^{*}$ \\
\hline 18 & Calyx lobe subequality & $\mathrm{C}$ & $\mathrm{mm} ;|\# 16-\# 17|$ \\
\hline 19 & Shape of tip of calyx segments & $\mathrm{N}$ & 5 \\
\hline \multicolumn{4}{|l|}{ Corolla } \\
\hline 20 & Total length & $\mathrm{C}$ & $\mathrm{mm}$ \\
\hline 21 & Teeth to bottom of corolla & $\mathrm{C}$ & $\mathrm{mm}^{*}$ \\
\hline 22 & Sinus of beak and lower lip to bottom & $\mathrm{C}$ & $\mathrm{mm} *$ \\
\hline 23 & Tube length & $\mathrm{C}$ & $\mathrm{mm} *$ \\
\hline 24 & Lower lip pouchy & $\mathrm{D}$ & $2 *$ \\
\hline 25 & Teeth petaloid & $\mathrm{D}$ & $2 *$ \\
\hline 26 & Stigmas exserted & $\mathrm{D}$ & $2 *$ \\
\hline 27 & Length of beak & $\mathrm{C}$ & $\mathrm{mm} ; \# 20$ - \#22* \\
\hline 28 & Beak length to tube length ratio & $\mathrm{C}$ & ratio; \#20 / \#27 \\
\hline
\end{tabular}




\section{Table 2 (on next page)}

Results of fuzzy clustering analyses.

Results of fuzzy clustering analyses with $\mathrm{k}=4,3$, and 2 clusters. Here we report average silhouette coefficients within and across clusters in analyses, as well as normalized Dunn coefficients for each analysis. Silhouette coefficients close to 0 represent less similarity, those close to 1 represent high similarity, and negative silhouette coefficients indicate likely misassignment to a cluster. The normalized Dunn coefficient is a measure of the overall fuzziness of an analysis. Values close to 0 indicate high levels of fuzziness (near equal membership of individuals to all clusters) and values close to 1 indicate very low levels of fuzziness (i.e., hard partitions). 


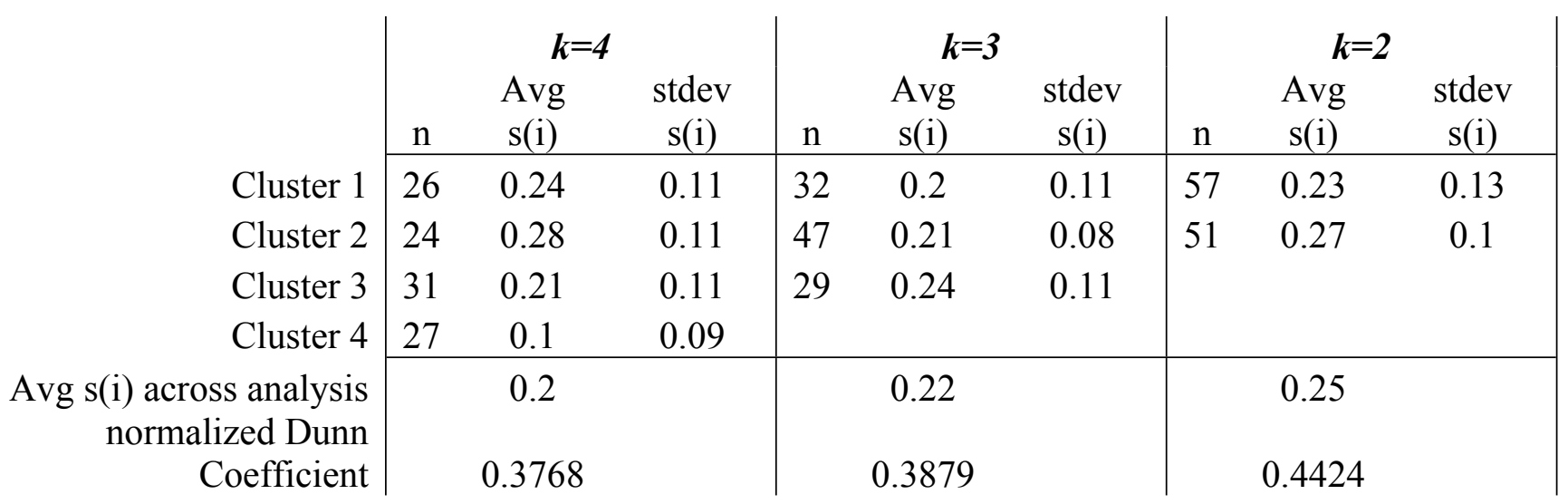

1 